\title{
Perfil epidemiológico del cáncer de próstata etapa clínica T1c en Oncólogos del Occidente de Caldas (Colombia)
}

\author{
- C. R. Villegas Mejía, J. A. Chacón Cardona, T. Sánchez Villegas, G. A. Rojas Uribe
}

Objetivo. Analizar el perfil epidemiológico de pacientes con cáncer de próstata en subetapa clínica T1C.

Método. Análisis descriptivo de los pacientes con diagnóstico de cáncer de próstata que asisten a Oncólogos del Occidente con una base inicial de 404 casos desde enero de 2007 a junio de 2012, y de ellos 136 presentaban una subetapa T1C, definida como el diagnóstico por biopsia en pacientes con enfermedad no palpable.

Resultados. La presentación por año de diagnóstico ha venido en aumento. La media de edad fue de 70,20 (SD: 8,1). Al diagnóstico se encontró etapa IIA y IIB en el $74 \%$. Con Gleason score menor a 6 en el $49 \%, 7$ en el $29 \%$ y entre $8-10$ en el $20 \%$. Media de PSA de 25,5 (SD: 48,7 ). El $60 \%$ sin tratamiento previo. El promedio de tiempo de síntomas previos fue de 7,1 meses (SD: 14,2 meses) con el
$65 \%$ de menos de tres meses de síntomas; el $98 \%$ de los pacientes con Karnofsky mayor al $80 \%$. Se presentó un 34\% de recaída con una media de 43,7 meses (SD: 34,1$)$ y, de ellos, el $78 \%$ con recaída bioquímica. En el $77 \%$ de pacientes no hubo cirugía. El $89 \%$ recibió radioterapia y, de ellos, el $71 \%$ al tumor primario. La supervivencia a 10 años es influenciada por la mayor edad al diagnóstico, el estadio, la clasificación más alta de Gleason, el nivel más alto de PSA, el nivel de Karnofsky obtenido y el tipo de cirugía previa.

Conclusión. Se presenta un panorama amplio del patrón de ocurrencia del cáncer de próstata localizado T1c y su relación con la sobrevida según diferentes factores pronósticos referidos en la literatura como un esfuerzo para aplicarlos en un área específica de Colombia y servir de referencia a otras zonas de similares características geográficas.

\section{Carcinoma escamocelular de tráquea metastásico: reporte de caso}

\section{- Giovanna P. Rivas ${ }^{1,2,3}$, Carlos E. Bonilla ${ }^{1,2}$, Alfonso Lozano ${ }^{3}$}

${ }_{1}^{1}$ Grupo de Oncología Clínica, Instituto Nacional de Cancerología, ESE, Bogotá (Colombia).

2 Universidad El Bosque.

${ }^{3}$ Centro Integral de Cáncer, Clínica de Occidente, Cali.

${ }^{4}$ Grupo de Radiología, Instituto Nacional de Cancerología, ESE, Bogotá (Colombia).

El carcinoma escamocelular de tráquea es un tumor raro, de comportamiento agresivo. La cirugía es el tratamiento de elección. La radioterapia se ha empleado en el escenario adyuvante o como tratamiento paliativo, mientras que el papel de la quimioterapia no está bien definido.

Se presenta el caso de un hombre de 33 años diagnosticado con carcinoma escamocelular de tráquea estadio IV con compromiso metastásico pulmonar y ganglionar mediastinal. Inicialmente fue llevado a manejo quirúrgico y radioterapia; luego recibió tratamiento con quimioterapia basada en carboplatino con intención paliativa. El paciente presentó progresión pulmonar, por lo que se inició terapia sistémica con platino más gemcitabina, logrando enfermedad estable; posteriormente presenta de nuevo progresión pulmonar y fallece; sin embargo, a pesar de lo agresivo de la enfermedad, logra 30 meses de supervivencia global. 